\title{
Phytoprotection
}

\section{Effet de la brûlure cercosporéenne, causée par le Cercospora carotae, sur le développement, la croissance et le rendement de la carotte \\ Effet of Cercospora blight, induced by Cercospora carotae, on development, growth and yield of carrot}

\author{
G. Bourgeois, C. Brodeur et A.C. Kushalappa
}

Volume 79, numéro 1, 1998

URI : https://id.erudit.org/iderudit/706131ar

DOI : https://doi.org/10.7202/706131ar

Aller au sommaire du numéro

Éditeur(s)

Société de protection des plantes du Québec (SPPQ)l

ISSN

0031-9511 (imprimé)

1710-1603 (numérique)

Découvrir la revue

Citer cet article

Bourgeois, G., Brodeur, C. \& Kushalappa, A. (1998). Effet de la brûlure cercosporéenne, causée par le Cercospora carotae, sur le développement, la croissance et le rendement de la carotte. Phytoprotection, 79(1), 9-19. https://doi.org/10.7202/706131ar
Résumé de l'article

L'objectif de cette étude était de quantifier l'effet de la sévérité de la brûlure cercosporéenne (Cercospora carotae) sur le développement (stades végétatifs), la croissance (poids secs du feuillage et des racines, indice de surface foliaire) et le rendement de la carotte (Daucus carota var. sativa). Au cours d'expériences effectuées en sol organique durant les saisons 1990, 1991 et 1992, trois niveaux de sévérité ont été obtenus par des nombres différents d'applications de fongicides, à savoir aucune application, deux ou trois applications et une application à chaque semaine. Au cours des trois années, des différences entre les traitements ont été observées 60 jours après le semis pour la sévérité de la maladie, 80 jours après le semis pour le poids sec du feuillage et l'indice de surface foliaire, et 100 jours après le semis pour le poids sec des racines. Une augmentation du nombre d'applications a permis une augmentation du rendement total en carottes. Cependant, les applications hebdomadaires n'ont pas augmenté le rendement en carottes de classe régulière par rapport à deux ou trois applications pour l'ensemble de la saison. Il est donc possible de réduire le nombre d'applications de fongicides, tout en conservant un rendement économiquement acceptable. 


\title{
Effet de la brûlure cercosporéenne, causée par le Cercospora carotae, sur le développement, la croissance et le rendement de la carotte
}

\author{
Gaétan Bourgeois ${ }^{1}$, Carole Brodeur ${ }^{1}$ et Ajjamada C. \\ Kushalappa ${ }^{2}$
}

Reçu 1997-11-25; accepté 1998-07-20

PHYTOPROTECTION $79: 9-19$

L'objectif de cette étude était de quantifier l'effet de la sévérité de la brûlure cercosporéenne (Cercospora carotae) sur le développement (stades végétatifs), la croissance (poids secs du feuillage et des racines, indice de surface foliaire) et le rendement de la carotte (Daucus carota var. sativa). $\mathrm{Au}$ cours d'expériences effectuées en sol organique durant les saisons 1990, 1991 et 1992, trois niveaux de sévérité ont été obtenus par des nombres différents d'applications de fongicides, à savoir aucune application, deux ou trois applications et une application à chaque semaine. Au cours des trois années, des différences entre les traitements ont été observées 60 jours après le semis pour la sévérité de la maladie, 80 jours après le semis pour le poids sec du feuillage et l'indice de surface foliaire, et 100 jours après le semis pour le poids sec des racines. Une augmentation du nombre d'applications a permis une augmentation du rendement total en carottes. Cependant, les applications hebdomadaires n'ont pas augmenté le rendement en carottes de classe régulière par rapport à deux ou trois applications pour l'ensemble de la saison. II est donc possible de réduire le nombre d'applications de fongicides, tout en conservant un rendement économiquement acceptable.

[Effect of Cercospora blight, induced by Cercospora carotae, on development, growth and yield of carrot]

The objective of this study was to quantify the effect of Cercospora blight of carrot (Cercospora carotae) severity on development (vegetative stages), growth (dry weight of leaves and roots, leaf area index) and yield of carrot (Daucus carota var. sativa). In experiments conducted on organic soils during 1990, 1991 and 1992, three levels of disease severity were obtained by different number of fungicide applications : no application, two or three applications, and weekly applications. During the three years, significant differences were observed at 60 days after sowing for disease severity, at 80 days after sowing for the dry weight of leaves and leaf area index, and at 100 days after sowing for the dry weight of roots. An increase in the number of fungicide applications resulted in an increase in total yield at

1 Agriculture et Agroalimentaire Canada, Centre de recherche et de développement en horticulture, 430, boul. Gouin, Saint-Jean-sur-Richelieu (Québec), Canada J3B 3E6. Contribution $n^{\circ}$ 335/98.08.03R

2 Département de phytologie, Campus Macdonald de I'Université McGill, 21111, Lakeshore, Sainte-Anne-de-Bellevue (Québec), Canada H9X 3V9 
final harvest. However, weekly fungicide applications did not result in an increase in yield of carrots in the regular size classification. It is thus possible to maintain an acceptable economic yield even if the number of fungicide applications is reduced.

\section{INTRODUCTION}

La brûlure cercosporéenne de la carotte, maladie foliaire provoquée par le champignon Cercospora carotae (Pass.) Solheim, est la plus importante maladie de la carotte (Daucus carota L. var. sativa (Hoffm.) Arcang.) au Québec. On la retrouve dans la majorité des champs en culture (Arcelin et Kushalappa 1991). La brûlure cercosporéenne est également présente en Ontario et aux ÉtatsUnis (Thomas 1943). L'infection produit sur le feuillage une tache brun foncé ou grise à peu près ronde. Une zone de nécrose peut apparaître autour de la tache, causée par la sécrétion de cercosporine par le champignon. Enfin, en pourtour, on peut voir une zone de chlorose. Lorsque les conditions sont favorables au développement de la maladie, les lésions grandissent rapidement, se rejoignent, et occasionnent une diminution de la photosynthèse sur une grande surface ainsi qu'une défoliation prématurée. Les feuilles dont les pétioles ont des lésions se détachent facilement, ce qui pose des problèmes lors de l'arrachage mécanique qui requiert la présence de feuillage.

Les recommandations du Conseil des productions végétales du Québec (1987) sont de quatre ou cinq traitements fongicides à 7 à $12 \mathrm{j}$ d'intervalle, selon la fréquence des pluies, en commençant lorsque le feuillage des carottes a de 10 à $15 \mathrm{~cm}$ de hauteur. Or, I'application de fongicides est coûteuse et peut être nuisible ou même toxique pour l'environnement. L'élaboration de stratégies de lutte permettant une réduction du nombre $d$ 'applications de fongicides tout en optimisant le rendement de carotte serait un apport positif pour les producteurs, l'environnement et les consommateurs. Boivin et al. (1990) ont proposé des plans d'échantillonnage séquentiel binomial pour les carottes cultivées en sol organique au Québec. Cette technique permet de déterminer un seuil d'action pour débuter les premiers traitements fongicides contre la brûlure cercosporéenne. Carisse et Kushalappa $(1990,1992)$ ont développé un modèle de prévision de l'infection par le $C$. carotae. Ce système permet d'identifier les périodes d'infection en fonction de la température de l'air, l'humidité relative et la mouillure du feuillage.

Cependant, aucune donnée scientifique n'est disponible à savoir si la défoliation ou même la nécrose du feuillage provoquée par la brûlure cercosporéenne a un effet sur la croissance et le rendement de la carotte. Or, pour que les producteurs puissent rationaliser I'utilisation des fongicides sur les carottes, il est nécessaire de bien comprendre l'impact de la sévérité de cette maladie et de son évolution dans le temps sur les pertes de rendement et de qualité. L'objectif de ce projet est donc de quantifier, en comparant trois niveaux de sévérité, l'effet de la brûlure cercosporéenne sur le développement (stades végétatifs), la croissance (poids sec du feuillage et des racines, indice de surface foliaire) et le rendement de la carotte.

\section{MATÉRIEL ET MÉTHODES}

Les expériences ont été menées en sol organique à la ferme expérimentale d'Agriculture et Agroalimentaire Canada à Sainte-Clotilde (lat. $45^{\circ} 49^{\prime} \mathrm{N}$, long. $73^{\circ} 41^{\prime}$ O) durant les saisons 1990, 1991 et 1992 , sur 12 parcelles de $10 \mathrm{~m}$ de longueur comprenant chacune 18 rangs distancés de $60 \mathrm{~cm}$. La distance entre les parcelles était de $5 \mathrm{~m}$. La superficie totale du champ était de 40,6 m x $55 \mathrm{~m}$. 
Des carottes cv. Six Pak II y ont été semées le 25 mai 1990, le 4 juin 1991 et le 26 mai 1992 à raison de 100 grains par mètre. Il y a eu irrigation en 1991 et 1992 afin de permettre la germination des semences. Les applications de pesticides et de fertilisants ont été effectuées selon les recommandations du Conseil des productions végétales du Québec (1987), sauf en ce qui concerne les fongicides. Ainsi les insectes, spécialement le charançon de la carotte (Listronotus oregonensis (LeConte)) furent réprimés lorsque nécessaire par l'emploi de phosmet (0,0-dimethyle phosphorodithioate S-ester avec $\mathrm{N}$-(mercapto-methyl) phtalimide) à une dose de $1,125 \mathrm{~kg} \mathrm{~m}$. a. ha-1 (1990: 28 juin et 11 juillet; $1991: 6$ juillet; $1992: 19$ juin et 25 juin). Pour prévenir la compétition avec les mauvaises herbes, il y a eu sarclage et application de linuron (3(dichloro-3,4 phényl)-1-méthoxy-Nméthyl-1-urée) à une dose de 1,2 $\mathrm{L} \mathrm{ha}^{-1}$ en pré- et post-émergence. À chaque printemps, avant les semis, le sol a été fertilisé avec du 5-5-20 à raison de $2,0 \mathrm{~L} \mathrm{ha}^{-1}$.

Le dispositif expérimental consistait en quatre blocs complètement aléatoires et trois niveaux de sévérité qui ont été obtenus avec des nombres différents d'application de fongicide : i) sévérité élevée (aucune application de fongicide), ii) sévérité intermédiaire (deux ou trois applications de fongicide) et iii) sévérité faible (application de fongicide à chaque semaine débutant de 33 à $63 \mathrm{j}$ après le semis (JAS)). Le fongicide utilisé a été le mancozèbe [(éthylène bis-(dithiocarbamate) de manganèse) et ion zinc] à $1,69 \mathrm{~kg} \mathrm{ha}^{-1}$. Le chlorothalonil (tetrachloroisopthalonitrile) a été employé en 1990, pour contrer le développement du Sclerotinia sclerotiorum (Lib.) de Bary, à raison de 1,2 à $1,6 \mathrm{~L} \mathrm{ha}^{-1}$. Pour le niveau de sévérité le plus faible, les premiers traitements fongicides ont été appliqués 34,49 et 63 JAS pour 1990, 1991 et 1992, respectivement. Les traitements ont été répétés à intervalles de 6 à $11 \mathrm{j}$ selon les conditions météorologiques et ont pris fin à la troisième semaine de septembre. Les traitements pour le niveau de sévérité intermédiaire ont eu lieu les 8 et 29 août, et le 12 septembre en 1990; les 22 et 30 août en 1991; et les 28 juillet et 27 août en 1992. Les applications ont été effectuées au moyen d'un épandeur de type M-SP 425 (Pulvérisation MS inc., Drummondville (Québec), Canada) avec buses à jets balai (modèle 8002) à une pression de $207 \mathrm{kPa}$.

\section{Analyse de croissance}

Des récoltes ont été effectuées à chaque semaine ou à toutes les deux semaines selon le stade de la culture. À 12 reprises pendant la saison de croissance, et débutant 32 JAS, les carottes d'une section de $1 \mathrm{~m}$ ont été récoltées dans chacune des parcelles. Sur ces carottes, 15, 10, 8 carottes ont été prélevées au hasard selon qu'on se trouvait au début, au milieu et à la fin de la saison, respectivement. Pour chacune de ces carottes, le stade, la longueur et le poids frais de la racine, le diamètre du collet et la hauteur des feuilles ont été mesurés. Puis, les poids secs des racines échantillonnées, des racines et des feuilles totales du mètre ont été évalués. Les feuilles de l'échantillon ont été classées en trois groupes : bas ( $\leq 15 \mathrm{~cm}$ de hauteur), milieu ( $>15 \mathrm{~cm}$ et $\leq 30 \mathrm{~cm}$ ) et haut $(>30 \mathrm{~cm}$ ) (Plaut et Berger 1980). La surface foliaire, le poids sec des feuilles et des tiges ont ensuite été mesurés pour chacun des trois groupes. La surface foliaire a été mesurée à I'aide d'un planimètre (LI-COR, modèle LI-3100). Tous les échantillons ont été séchés à $60^{\circ} \mathrm{C}$ jusqu'à ce qu'aucun changement de masse ne soit perceptible, puis le poids sec fut déterminé. Aux mêmes dates, nous avons suivi le développement de plantes préalablement choisies en marquant toute nouvelle feuille étalée et en prenant note de la défoliation.

\section{Évolution de la maladie}

La sévérité de la maladie a été déterminée dans chacune des parcelles à chaque récolte. Pour ce faire, le pourcentage de surface foliaire atteinte a été évalué au moyen de l'échelle améliorée de Horsfall-Barratt (1945), sur un échantillon de huit feuilles du bas, huit du milieu et huit du haut; d'autre part, le suivi minutieux du développement des 
plantes (numérotation des nouvelles feuilles) a permis de détecter la mort de chacune des feuilles. Dans les parcelles expérimentales, la défoliation observée chez la carotte pouvait être naturelle, surtout au niveau des feuilles plus âgées, ou induite par la brûlure cercosporéenne. La défoliation induite par la maladie a été obtenue en comparant, pour chaque bloc, la surface foliaire de chaque parcelle avec celle du niveau de sévérité faible où seulement une défoliation naturelle était observée. La sévérité de la maladie $\left(s_{\mathrm{y}}\right)$ a été déterminée par l'expression de la proportion de défoliation induite par la maladie $\left(d_{t}\right)$ combiné avec la proportion de surface foliaire nécrosée $\left(n_{t}\right)$ :

$$
s_{v}=n_{t}\left(1-d_{t}\right)+d_{t}
$$

\section{Effet sur les rendements}

À la fin de la saison, soit de 125 à 132 JAS, nous avons procédé à I'analyse commerciale. Pour évaluer l'effet de la brûlure cercosporéenne sur le rendement et la classification des carottes à la récolte, des carottes ont été récoltées manuellement sur deux segments de $3 \mathrm{~m}$ dans chacune des parcelles, le feuillage a été séparé et les carottes lavées afin d'enlever la terre. Les carottes ont été séparées dans les classes suivantes : petites (longueur $<11,5 \mathrm{~cm}$, diam $<1,9 \mathrm{~cm}$ ), régulières (longueur $>11,5 \mathrm{~cm}$, diam entre 1,9 et $3,8 \mathrm{~cm})$, jumbo (diam $>3,8 \mathrm{~cm}$ ) et autres (fourchues, croches, fendues, avariées). Le poids frais a été noté pour chacune des classes.

\section{Analyse des résultats}

La comparaison entre les traitements a été effectuée à I'aide du logiciel SAS (1987) au moyen de contrastes orthogonaux pour chacune des dates d'échantillonnage et pour la récolte finale. Les analyses statistiques visaient surtout à identifier le moment dans la saison de croissance où des différences significatives étaient observées. Les probabilités ont été considérées significatives à partir de $P \leq 0,05$. À l'occasion dans le texte, $P$ est spécifié pour des valeurs entre 0,06 et 0,10 .

\section{RÉSULTATS ET DISCUSSION}

À l'examen des données météorologiques, I'année 1991 fut une saison plus sèche lors de la période de développement de la maladie (mi-juillet à fin août). Or, l'effet de la pluie sur la dissémination de la maladie est bien documenté (Carisse et Kushalappa 1990, 1992). La pluie, pour les autres années, a été concentrée surtout en début de saison (mi-juin à fin juillet) en 1990 et généralisée au cours de la saison en 1992. A posteriori, nous constatons avoir obtenu un éventail de conditions météorologiques pour étudier la brûlure cercosporéenne.

Les premiers symptômes de la maladie (nécrose) ont été observés 62 JAS en 1990, 55 JAS en 1991 et 48 JAS en 1992 (fig. 1). Le pourcentage de surface foliaire nécrosée $n^{\prime}$ a jamais dépassé les $20 \%$ en 1990 et en 1991, mais en 1992, année particulièrement pluvieuse, il a atteint $38 \%$ dans le traitement à sévérité élevée, c'est-à-dire celui sans fongicide.

\section{Évolution de la maladie}

La sévérité de la maladie comporte deux variables : la défoliation et la surface nécrosée. Comme on peut le constater sur la figure 1 pour les parcelles non traitées en 1990, la courbe de sévérité de la maladie suit intimement celle de la défoliation. La même observation est valable pour les deux autres années. $C^{\prime}$ est donc dire que la plante ne supporte pas une grande quantité de nécrose avant que la défoliation ne soit induite. Ainsi, les cultivars qui génèrent beaucoup de feuillage seront avantagés face à la brûlure cercosporéenne et particulièrement en fin de saison.

Le premier facteur pour lequel on a noté une différence significative fut évidemment la sévérité de la maladie (fig. 2 à 4). Le traitement à sévérité faible s'est démarqué des deux autres dès 62 , 55 et 91 JAS pour 1990, 1991 et 1992 respectivement. Pour noter des écarts 

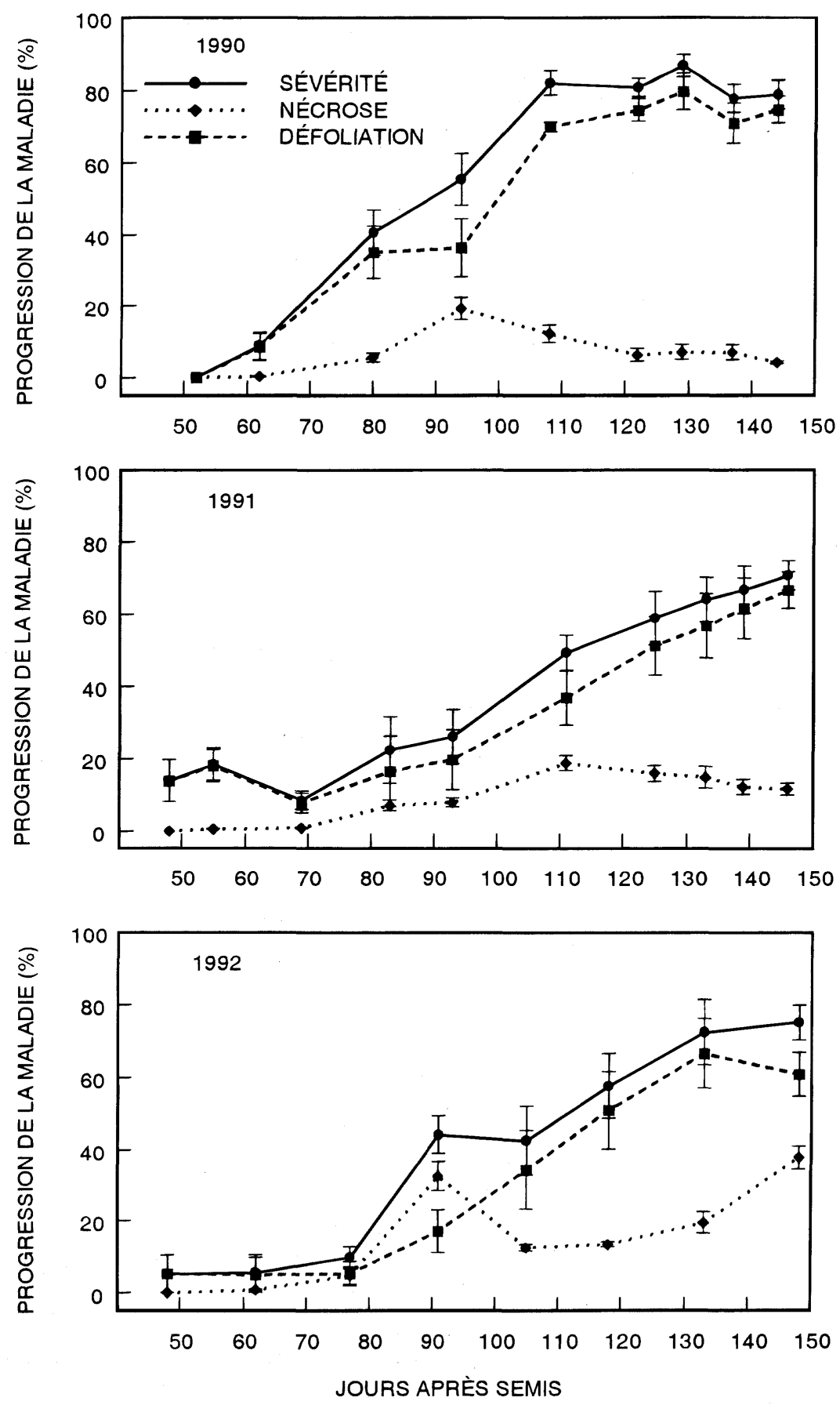

Figure 1. Progression de la maladie en termes de sévérité, défoliation et surface foliaire nécrosée pour les parcelles non traitées en 1990, 1991 et 1992. 

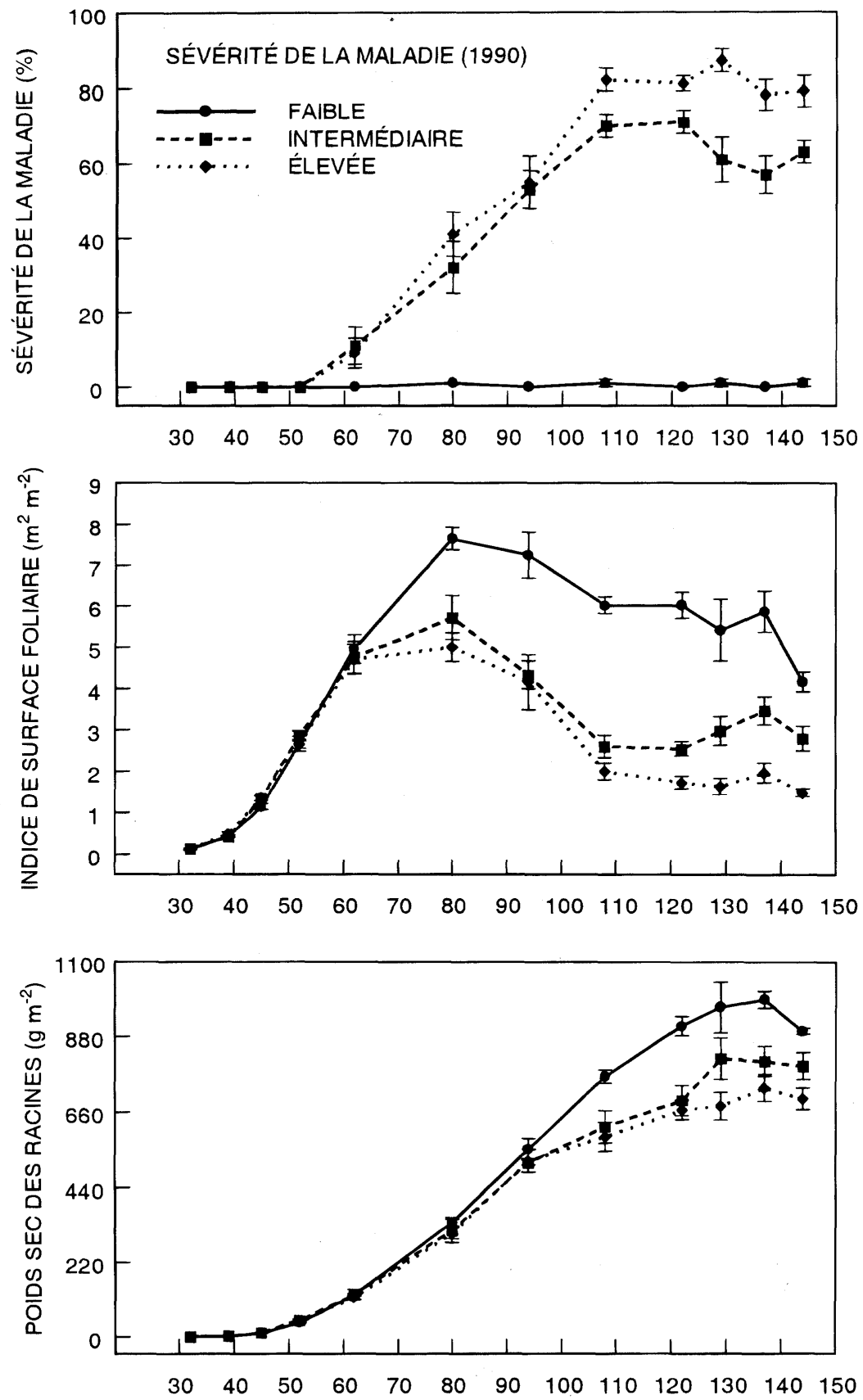

JOURS APRÈS SEMIS

Figure 2. Décalage dans le temps des courbes de trois paramètres : sévérité de la maladie, indice de surface foliaire et poids sec des racines, en 1990, pour les différents niveaux de sévérité de la maladie (sévérité faible, intermédiaire et élevée). 

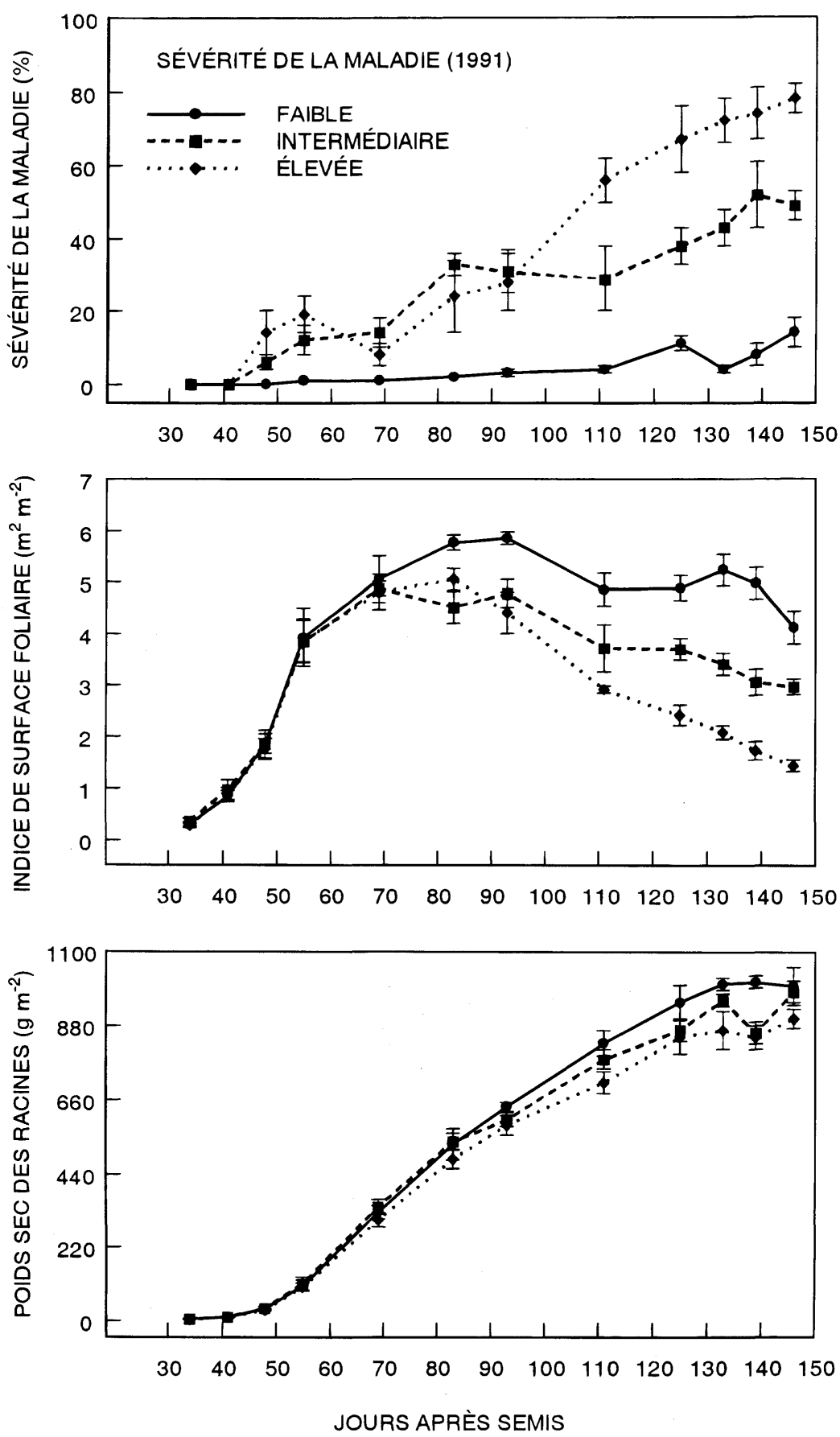

Figure 3. Décalage dans le temps des courbes de trois paramètres : sévérité de la maladie, indice de surface foliaire et poids sec des racines, en 1991, pour les différents niveaux de sévérité de la maladie (sévérité faible, intermédiaire et élevée). 

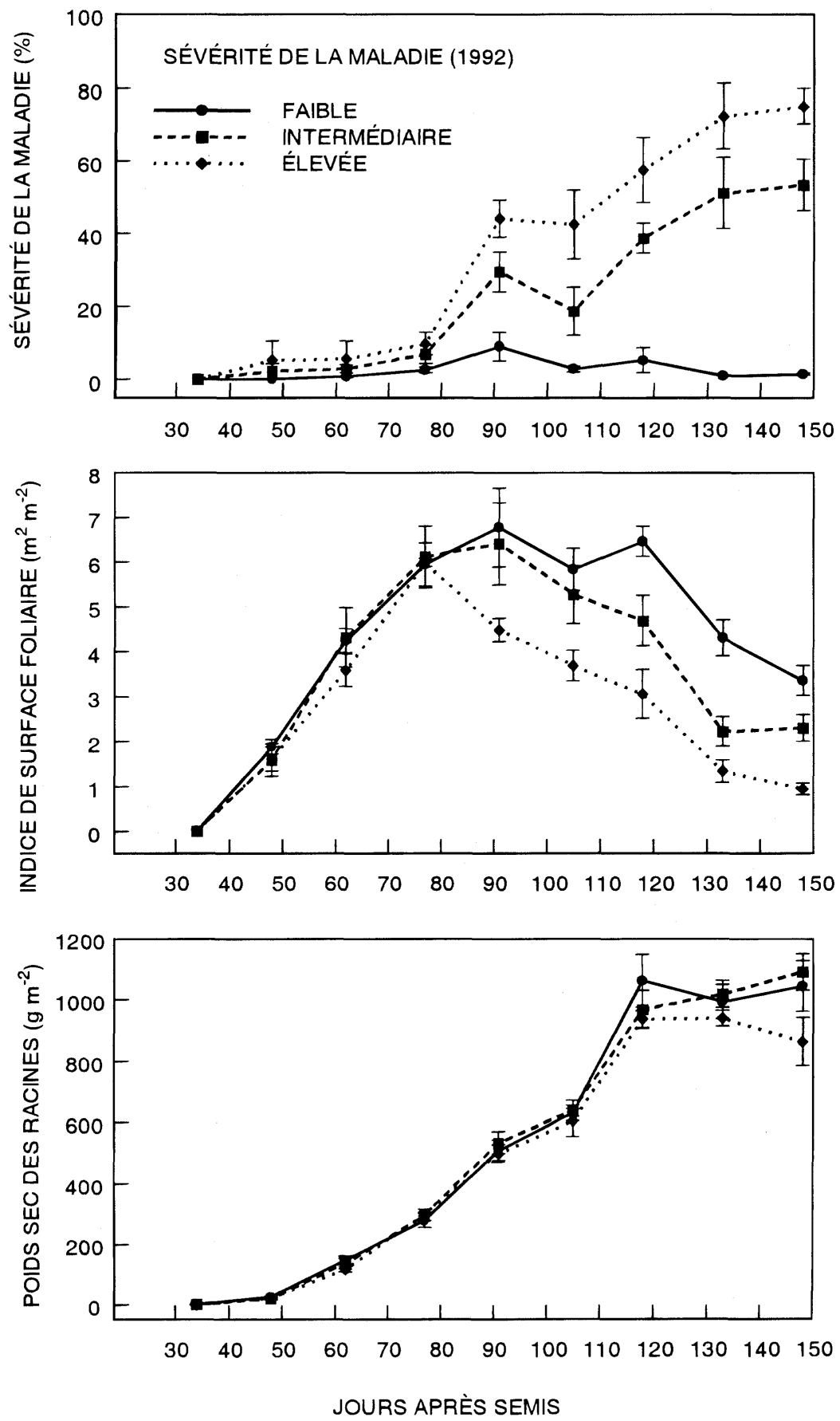

Figure 4. Décalage dans le temps des courbes de trois paramètres : sévérité de la maladie, indice de surface foliaire et poids sec des racines, en 1992, pour les différents niveaux de sévérité de la maladie (sévérité faible, intermédiaire et élevée). 
entre les traitements à sévérité intermédiaire et élevée, il a fallu attendre, dans l'ordre habituel, 108, 111 et 91 JAS, au seuil $P \leq 0,06$ pour 1991 et 1992 .

La différence entre les traitements a été fortement accentuée en ce qui concerne la nécrose seulement. Le traitement à sévérité faible s'est différencié des deux autres traitements à partir de 80,83 et 91 JAS pour les trois années respectivement. Les deux autres traitements ne se sont, par contre, jamais distingués I'un de l'autre $(P>0,10)$. Ce dernier résultat était prévisible puisque la nécrose est une variable trop volatile pour qu'un écart soit possible, surtout en fin de saison. De fait, la défoliation massive d'un plant peut le faire passer de très nécrosé à sain en quelques jours.

\section{Effet de la maladie sur la croissance de la plante}

Tel que prévu, le contraste entre les traitements en ce qui a trait à l'indice de surface foliaire (fig. 2 à 4) a suivi le même modèle que celui de la nécrose à cette différence près qu'en 1992, il a fallu attendre à 118 JAS pour observer un écart entre la sévérité faible et les autres traitements et que la sévérité intermédiaire s'est distinguée de la sévérité élevée après 137,125 et 148 JAS. La différence qu'on peut remarquer entre le traitement à sévérité intermédiaire et le témoin sans fongicide provient de la défoliation qui s'additionne à la nécrose pour réduire l'indice de surface foliaire. Cette dernière variable constitue donc un marqueur précieux et très sensible pour comparer différents traitements dans le cas de la brûlure cercosporéenne de la carotte où la défoliation survient facilement à la suite de nécrose. Notons que le poids sec du feuillage a suivi intimement la courbe de l'indice de surface foliaire.

\section{Effet sur les rendements en poids sec de racines}

L'écart entre le témoin sans maladie et les autres traitements en ce qui concerne le poids sec des racines est devenu significatif après $108 \mathrm{JAS}$ en 1990, mais les sévérités intermédiaire et élevée sont demeurées statistiquement semblables (fig. 2). Nous avons observé la même évolution du poids sec des racines pour
1991, la différence significative survenant à 93 JAS (fig. 3). En 1992, à part les nuances notées au cours de la saison, I'ANOVA n'a indiqué aucun écart significatif entre les trois niveaux de traitements jusqu'à la récolte pour toutes les dates d'échantillonnage (fig. 4). On peut tout de même observer une tendance, les poids secs des racines récoltées étant de 1045 et $1091 \mathrm{~g} \mathrm{~m}^{-2}$ pour les traitements à sévérités faible et intermédiaire respectivement et de $864 \mathrm{~g} \mathrm{~m}^{-2}$ pour les parcelles sans fongicides à la dernière date d'échantillonnage soit à 148 JAS, même si non-significatif.

Il est évident que si la brûlure cercosporéenne affecte de façon importante I'indice de surface foliaire, elle n'a qu'une répercussion réduite sur le rendement, tout au long de la saison. C'est la défoliation, beaucoup plus que la surface nécrosée des feuilles vivantes, qui est responsable de la perte de rendement, même si celle-ci demeure très réduite.

Comme on peut le voir sur les figures 2 à 4 , il existe une relation temporelle entre l'apparition de la maladie, la diminution de l'indice de surface foliaire puis la baisse de rendement de carottes. Tel que prévu, l'effet du $C$. carotae est d'abord perceptible par l'augmentation de la surface nécrosée de la plante. Puis on note une diminution de l'indice de surface foliaire et deux semaines plus tard, la réduction du poids sec des racines.

\section{Récolte finale et analyse commerciale}

L'hypothèse de base sous-tendant l'analyse de la qualité commerciale était que la sévérité élevée était différente des deux autres en ce qui a trait au rendement final.

Le rendement total en carottes, toutes classes confondues, fut significativement différent entre les trois traitements pour toutes les années d'expérimentation, à une exception près : en 1992, le traitement à sévérité intermédiaire a donné statistiquement autant de carottes que celui à sévérité faible $(9114 \mathrm{~g}$ $\mathrm{m}^{-2}$ contre $\left.10113 \mathrm{~g} \mathrm{~m}^{-2}, P \leq 0,1\right)$. Pour les conditions de marché, il est toutefois 

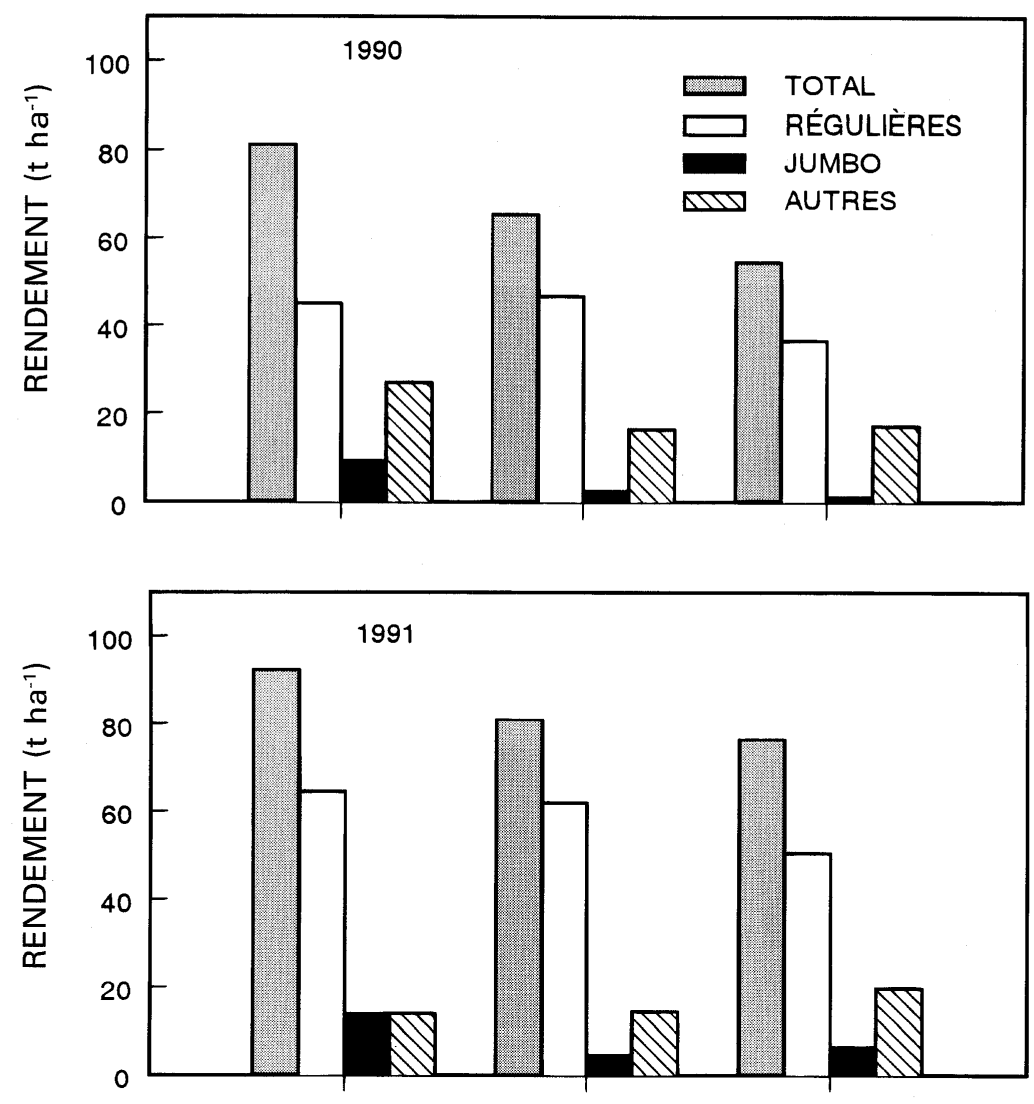

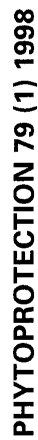

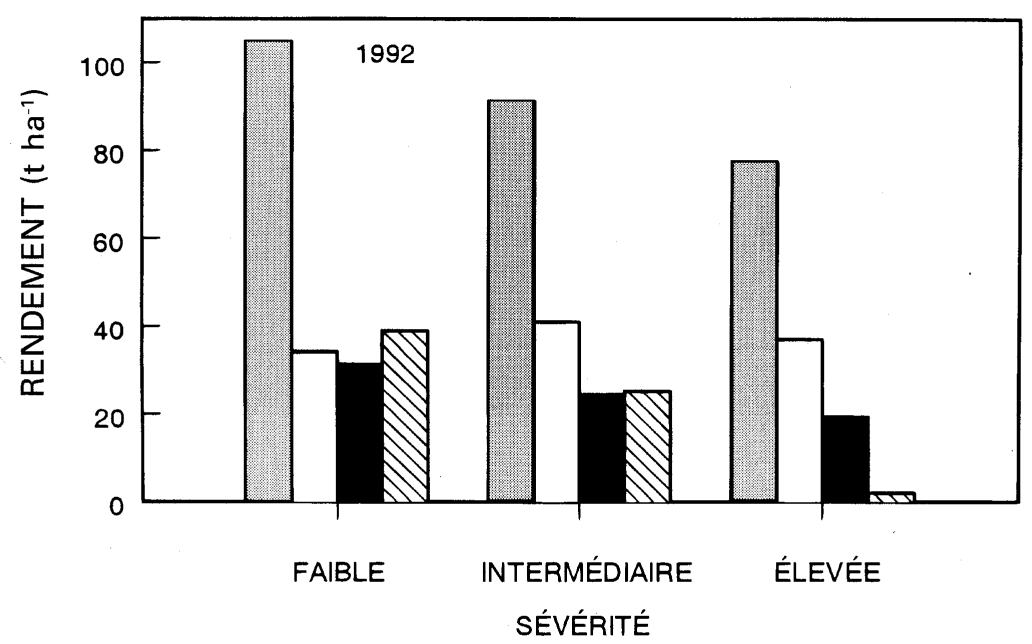

Figure 5. Rendements à la récolte pour chacun des traitements (sévérité faible, intermédiaire et élevée) en 1990, 1991 et 1992. 
plus intéressant d'analyser la classification des carottes.

En 1990, nous avons noté un écart significatif dans le rendement en carottes régulières entre les parcelles traitées et le témoin non-traité $(P \leq 0,01)$ (fig. 5). En poursuivant le contraste orthogonal, un tel écart $n$ 'existait pas entre les deux niveaux de traitements fongicides $(P \geq 0,5)$. II en a été de même en 1991 (respectivement $P \leq 0,01$ et $P \geq$ $0,5)$. Toutefois, en 1992, il $n^{\prime} y$ a eu aucune différence significative entre un ou l'autre des traitements $(P \geq 0,6)$. Dans tous les cas, les carottes régulières ont représenté plus de la moitié et parfois même plus des deux tiers de la production, sauf en 1992 où nous avons observé un minimum de $39 \%$ de carottes régulières dans le témoin sans maladie.

Les carottes de la catégorie «jumbo» ont présenté un écart significatif entre les sévérités intermédiaire et faible pour 1990 et 1991 mais l'écart significatif fut entre les parcelles traitées versus les non traitées en 1992. En observant les graphiques de la figure 5 , on peut remarquer une tendance : plus on traite, plus on récolte de «jumbo». Par contre, I'accroissement du rendement en «jumbo" peut se faire au détriment du rendement en régulières, comme ce fut le cas en 1992.

Comme il y a peu ou pas de différence entre les sévérités faible et intermédiaire, il est donc possible de réduire substantiellement les applications fongicides sans affecter le rendement en carottes régulières. II peut même, dans certains cas, être avantageux de supporter un peu de maladie pour réduire la production de jumbo qui s'effectue au détriment des régulières.

Il est possible de réduire la fenêtre de traitements en commençant plus tard et en cessant plus tôt. Par exemple, vers la fin de la saison, lorsque les conditions de température deviennent défavorables pour la brûlure cercosporéenne, on peut cesser les traitements mais on court le risque d'exposer son champ à la brûlure alternarienne (Arcelin et Kushalappa 1991). Et enfin, dans le cas des carottes hâtives, puisqu'elles sont semées plus tôt et qu'elles sont récoltées à 100 ou 110 JAS, il est possible de ne pas traiter du tout, surtout lorsque les conditions ne favorisent pas la brûlure cercosporéenne.

\section{REMERCIEMENTS}

Cette recherche a été réalisée en partie grâce au financement d'Agriculture Canada dans le cadre d'une Entente auxiliaire Canada-Québec sur le développement agro-alimentaire. Nous tenons à remercier Mme Dominique Plouffe-Tellier et M. Mario Boivin pour leur collaboration technique, ainsi que le personnel de la ferme expérimentale d'Agriculture et Agroalimentaire Canada à Sainte-Clotilde.

\section{RÉFÉRENCES}

Arcelin, R. et A.C. Kushalappa. 1991. A survey of carrot diseases on muck soil in the southwestern part of Québec. Can. Plant Dis. Surv. 71 : 147-153.

Boivin, G., A.C. Kushalappa et L. Brodeur. 1990. Spatial dispersion and binomial sequential sampling plan for Cercospora carotae on carrots. Can. J. Plant Pathol. $12: 209-212$.

Carisse, O. et A.C. Kushalappa. 1990. Development of an infection model for Cercospora carotae on carrot based on temperature and leaf weatness duration. Phytopathology 80 : 1233-1238.

Carisse, O. et A.C. Kushalappa. 1992. Influence of interrupted wet periods, relative humidity and temperature on infection of carrots by Cercospora carotae. Phytopathology 82 : 602-606.

Conseil des productions végétales du Québec (CPVQ). 1987. Légumes-protection. Ministère de I'Agriculture, des Pêcheries et de l'Alimentation du Québec, Québec, Canada. 112 pp.

Horsfall, J.G. et R.W. Barratt. 1945. An improved grading system for measuring plant diseases. Phytopathology $35: 655$ (résumé).

Plaut, J.L. et R.D. Berger. 1980. Development of Cercosporidium personatum in three peanut canopy layers. Peanut Sci. 7 : 46-49.

SAS Institute Inc. 1987. Guide for personal computers. Version 6 edition. SAS Institute Inc., Cary, NC. 1028 pp.

Thomas, H.R. 1943. Cercospora blight of carrot. Phytopathology 33 : 114-125. 